\title{
The Peer Review Process During the COVID-19 Pandemic
}

Reid A. Paul, MA; Joyce Brody; and Cynthia M.A. Geppert, MD

\begin{abstract}
Reid Paul is Editor, Joyce Brody is Managing Editor, and Cindy Geppert is Editor in Chief. Correspondence: Reid Paul

(rpaul@mdedge.com)
\end{abstract}

Fed Pract. 2021;38(7). Published online July 11 doi:10.12788/fp.0157
T he COVID-19 pandemic put unparalleled strain on US health care systems and individual health care providers (HCPs), which has been well documented. Like all other medical peer reviewed journals, Federal Practitioner relies heavily on the generosity and dedication of federal HCPs. As the pandemic unfolded, we questioned whether HCPs would have the time and energy to write new articles, complete research projects, and review the work of their peers. To assess the impact of COVID-19 on the journal, we compared data from a full year during the COVID-19 pandemic with that of the previous year to determine whether and how the pandemic reshaped the peer review and publication process.

For the purposes of this review, we will compare a full year of COVID-19 journal performance with the prior year. Since COVID-19 infections spiked at different times in different places, there is no clear starting point for the pandemic. Similarly, states varied widely in their vaccination rates and opening procedures. Nevertheless, the period from May 1, 2020 to April 30, 2021, most of the country experienced COVID-19 restrictions, and the number of cases rose dramatically.

From May 1, 2020 to April 30, 2021, Federal Practitioner received 208 submissions, 110\% increase over the previous year (189 submissions from May 1, 2019 to April 30, 2020) and a $28 \%$ increase over a 2 -year period. After submission, it took an average of 9.0 days to the first reviewer invitation compared with 10.3 days in the previous year and 4.7 days 2 years prior. Time from the initial submission to the first decision (ie, accept, reject, or revise) took 72.8 days in the COVID-19 year compared with 91.1 days in the previous year and 69.6 days 2 years prior. In both periods it took reviewers a mean 9.5 days to complete a review from the date invited, and the rate of late reviews was unchanged as well.
During the COVID-19 pandemic year, 1481 reviewer invitations were sent to potential reviewers and 498 reviews were completed (33.6\%) by 195 individual reviewers: an average of 2.4 reviews per manuscript. Most reviewers recommended to accept the manuscript, and just $14.7 \%$ of reviewers recommended to reject the manuscript (Table). The previous year 1295 invitations were sent to potential reviewers and 460 reviews were completed (38.1\%) by 181 individual reviewers for an average of 2.4 reviews per manuscript.

For the original submissions, the journal accepted just $26(12.7 \%)$ articles, recommended revisions for 105 (51.2\%) submissions, and rejected 74 (36.1\%) submissions from May 1 , 2020 to April 30, 3021. One hundred seven manuscripts were revised once, and $75.7 \%$ were accepted, and $2.8 \%$ were rejected. Twentytwo articles had a second revision and 1 had a third revision and all were published. In the year before the pandemic, just 16 (9.5\%) manuscripts were accepted in their original form and 59 (39.1\%) were rejected.

Federal Practitioner published 113 articles from May 2020 to April 2021. These articles included 44 (38.9\%) original studies, 25 (22.1\%) case studies, $20(17.7 \%)$ program profiles, $16(14.2 \%)$ commentaries/editorials, and $8(7.1 \%)$ review articles; $19(16.8 \%)$ articles were focused on COVID-19. The prior year saw Federal Practitioner publish 106 articles in 18 issues. Of these articles $36.8 \%$ were original studies, $22.6 \%$ were program profiles, $18.9 \%$ were case studies, $13.2 \%$ were commentaries/editorials, and $8.5 \%$ were review articles.

Despite the impact of COVID-19, federal HCPs continued to contribute to this journal without significant interruption. The journal saw a $10 \%$ increase in submissions during the pandemic year compared with the previous year but that was in keeping with prior increases in submissions. Similarly, the journal saw more 
TABLE Peer Review Process Results

\begin{tabular}{lccccccc} 
& & \multicolumn{2}{c}{ Reviewers } & \multicolumn{3}{c}{ Journal Decisions } \\
\cline { 3 - 8 } Period & $\begin{array}{c}\text { Submissions, } \\
\text { No. }\end{array}$ & $\begin{array}{c}\text { Invitations } \\
\text { Sent, No. }\end{array}$ & $\begin{array}{c}\text { Reviews, } \\
\text { No. }\end{array}$ & $\begin{array}{c}\text { Time, } \\
\text { d }\end{array}$ & $\begin{array}{c}\text { Accept, } \\
\text { No. (\%) }\end{array}$ & $\begin{array}{c}\text { Reject, } \\
\text { No. (\%) }\end{array}$ & $\begin{array}{c}\text { Revise, } \\
\text { No. (\%) }\end{array}$ \\
\hline May 2020 to April 2021 & 208 & 1481 & 498 & 72.8 & $26(12.7)$ & $74(36.1)$ & $105(51.2)$ \\
\hline May 2019 to April 2020 & 189 & 1295 & 460 & 91.1 & $16(9.5)$ & $59(39.1)$ & $93(51.4)$
\end{tabular}

individual reviewers submit more total reviews from May 2020 to April 2021 compared with the previous year. The broad spectrum of reviewers involved in the process and the growing volume of both reviews and submissions suggest that our reviewers remained available and committed to the peer review process despite the impact of a pandemic.

Reducing the time to first decision remains an important priority for the journal. Although the time was shortened during the pandemic, it still took longer to inform authors of the first decision compared with 2 years before. There is no indication that COVID-19 had an impact on the speed of decision making. Reviewers were as timely during the pandemic as they were the year before.

Similarly, there was little difference in the types of articles that were published, other than the obvious increase in COVID-19 submissions. Most of the articles on COVID-19 were editorials and columns, though the journal also published case studies, program profiles, and review articles on treatment. During the pandemic, a higher percentage of articles were original studies and case reports, and fewer were program profiles compared with the types the year before. It is unclear if these differences resulted from random fluctuations in unsolicited manuscripts or are part of a larger trend. The journal managed to publish slightly more articles from May 2020 to April 2021 compared with May 2019 to April 2020 despite fewer issues. This is likely due to increased submissions and articles published online.

For the original submissions, the journal accepted just $26(12.7 \%)$ articles, recommended revisions for 105 (51.2\%) submissions and rejected 74 (36.1\%) submissions from May 2020 to April 3021. One hundred seven manuscripts were revised once and $75.7 \%$ were accepted and $2.8 \%$ were rejected. Twenty-two articles had a second revision and 1 had a third revision and all were published. In the year prior to the pandemic, just 16 (9.5\%) manuscripts were accepted in their original form, and 59 (39.1\%) were rejected.

Although Federal Practitioner improved the efficiency of its decision making, there is still significant room for improvement. We are committed to providing our authors with more rapid decisions and reducing the time to the first decision. Seventy-two days is still too long for authors to wait to hear about the initial decision on their article. Future reviews of the publication process should focus not only on the types of articles that are included, but their subjects as well. Given the great diversity of clinical care practiced across the US Department of Veterans Affairs, US Department of Defense, and the US Public Health Service, the journal must ensure that its articles reflect its diverse audience. We would like to see articles come from authors associated with all 3 major branches of our audience, as well as small portions of the readership (eg, Federal Bureau of Prisons, National Institutes of Health) and ask our readers to help us promote the journal to potential authors in all Federal Health Care organizations. We are especially interested in submissions on or from underserved populations.

Despite the significant burdens on HCPs and federal health care systems, Federal Practitioner managed to increase the speed of publication and the number of articles between May 2020 and April 2021 thanks to the work of all the authors and reviewers who contributed their time and energy to the publication during this challenging period. Their efforts are impressive and greatly appreciated. We pledge to continue to improve our process to reduce the time to publication and to continue to provide regular updates on our process and performance. 